https://doi.org/10.15407/ujpe64.10.962

S.I. MELNYK, S.S. MELNYK, A.A. LAVRINOVICH, M.T. CHERPAK

O.Ya. Usikov Institute for Radiophysics and Electronics, Nat. Acad. of Sci. of Ukraine

(12, Academician Proskura Str., Kharkiv 61085, Ukraine; e-mail: cherpak@ire.kharkov.ua)

\title{
TO THE PHENOMENOLOGICAL THEORY OF AVALANCHE-LIKE EFFECT IN THE DC-BIASED MICROWAVE NONLINEAR HTSC-BASED TRANSMISSION LINE
}

\section{Introduction}

Nonlinear effects in the microwave physics of condensed matter, which emerge when the medium is subjected to the action of electromagnetic fields, are of great interest to physicists and research engineers. A large body of works was devoted to microwave nonlinear phenomena in high-temperature superconductors (HTSCs) [1-4]. In particular, HTSC specimens with various compositions and in various electrodynamic structures were studied. As a rule, those structures were fabricated in the form of microwave resonators or transmission lines on the basis of epitaxial HTSC films.

When experimentally studying the properties of a microwave transmission line of the coplanar waveguide (CPW) type based on HTSCs, strong (jumplike) changes in microwave power losses were revealed at certain values of input power $P_{\mathrm{in}}$, dc current $I_{\mathrm{dc}}$, and waveguide temperature $T<T_{\mathrm{c}}$, where $T_{\mathrm{c}}$ is the critical temperature $[5,6]$. In this connection, a number of works should be mentioned, in which the transition of microwave HTSC-based structures into the dissipative state also occurred at certain values of a

(C) S.I. MELNYK, S.S. MELNYK, A.A. LAVRINOVICH, M.T. CHERPAK, 2019 direct current, and the microwave signal was intentionally weak, being used only to control a state of the HTSC structure (see, e.g., work [7]).

Anyway, there is no rigorous theoretical model to describe the features of the nonlinear HTSC-based transmission line. The well-known phenomenological model for the propagation of a microwave signal through the transmission line [8-10] does not take the presence of the dc component into account. Therefore, in this work, in order to quantitatively describe the observed effect, a task was formulated to generalize the known phenomenological model onto the case of power supply to the microwave transmission line by a direct current. A key point was to elucidate the possibility of accurate measurements of power losses in the transmission line in a highly dissipative state, in which the researched object can remain for a very short time (a few seconds) and which can result in its thermal destruction.

It turned out that the character of the analyzed dependence allows the jump-like changes in the waveguide properties to be considered as "catastrophes", e.g., of the fold type [11], and the methodological and mathematical apparatus of the theory of catastrophes to be applied in order to predict new results. The latter include the phenomenon of hysteresis in the tem-

ISSN 2071-0194. Ukr. J. Phys. 2019. Vol. 64, No. 10 
perature dependence of microwave losses, which can be confirmed in further experiments.

We may also presume that there is a possibility to reproduce the critical temperature of the " jump" at a given direct current - or, vice versa, the critical current at a constant temperature, and so forth - with a high accuracy. In principle, those effects may make it possible to create of high-precision microwave power, temperature, and dc sensors on the basis of microwave HTSC-based transmission lines, in particular, CPWs.

\section{Preliminary Analysis of Experimental Results}

Figure 1 illustrates the schematic diagram of the CPW cross-section [5]. The CPW was fabricated (THEVA, Germany) using the photolithographic method on the basis of an epitaxial HTSC film $\mathrm{YBa}_{2} \mathrm{Cu}_{3} \mathrm{O}_{7-\delta}\left(T_{\mathrm{c}}=86.5 \mathrm{~K}, I_{\mathrm{c}}=3.6 \times 10^{6} \mathrm{~A} / \mathrm{cm}^{2}\right.$ at $\mathrm{T}=77 \mathrm{~K}$ ) deposited onto a $\mathrm{MgO}$ single crystalline substrate. The dimensions of the CPW cross-section (see Fig. 1) were $a=0.186 \mathrm{~mm}, w=0.1 \mathrm{~mm}$, and $h=0.5 \mathrm{~mm}$. The CPW was a straight section of the length $l=16.81 \mathrm{~mm}$ with gold-covered contact pads, through which the microwave signal was supplied and received, and the direct current was transmitted (through the central conductor) using a microwave planar branch box.

The dependences of microwave losses $I L=$ $=10 \lg \left(P_{\text {out }} / P_{\text {in }}\right)$ on the temperature were experimentally studied for various values of the input microwave power $P_{\text {in }}$ and the magnitudes of the direct current through a waveguide. Typical relevant dependences measured in the presence and absence of the direct current can be found in work [5].

The experiment was carried out only in the temperature growth regime, which was slow enough for the thermodynamic equilibrium to be established in a waveguide. The time of the HTSC structure exposure to microwave radiation (current) was determined by a pulse duration of $5 \mu \mathrm{s}$ and a pulse repetition frequency of $2.5 \times 10^{4} \mathrm{~Hz}$. Those parameters enabled us to neglect the overall heating of the HTSC film during measurements. The direct current magnitude was always below its critical value.

It was shown that, in the absence of a direct current, the effect of a more drastic increase of $I L$ was also observed at a temperature of 84-86 K. As a working hypothesis, we may suppose that the direct cur-

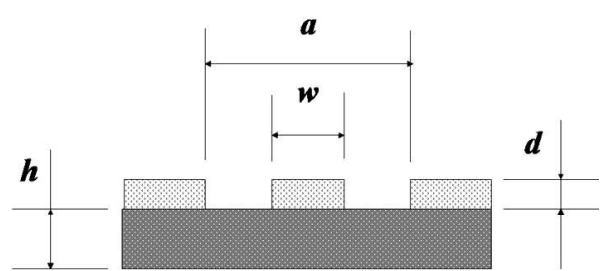

Fig. 1. Schematic diagram of the cross-section of a HTSCbased coplanar waveguide

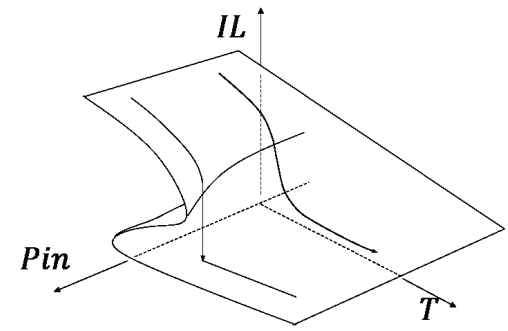

Fig. 2. The space of states of the system can look like a "fold" that emerges at the two-dimensional surface $I L\left(P_{\text {in }}, T\right)$

rent makes the temperature dependence of $I L$ even steeper and, at some "critical" current value, invokes an "avalanche-like" transition of the structure into a highly dissipative state, in which the losses are 2-3 orders of magnitude larger.

A system behavior of this kind is called a "catastrophe" [11]. Provided that the value $I_{d c}$ of the direct current through a waveguide is fixed, the system state is governed by two control parameters: the microwave input power $P_{\text {in }}$ and the film temperature $T$. The state variable $I L$, which depends on them, forms a two-dimensional response surface. It can be obtained as a solution of corresponding equations. In the general case, a "catastrophe" event arises at the boundary of a two-dimensional region, where the solution of equations becomes ambiguous (a bifurcation set). In one of the simplest cases, the corresponding solution has a character of a "fold" that arises in the three-dimensional space (Fig. 2).

Experimental studies of the dependence $I L\left(P_{\mathrm{in}}, T\right)$ showed that the "fold" can emerge even in the absence of a direct current component (Fig. 3). However, in this case, the bifurcation set of control parameters is empty, so that no "catastrophe" is observed.

Thus, the observed effect can be explained, e.g., by a deformation of the response surface $I L\left(P_{\text {in }}, T\right)$ at a nonzero direct current value and due to the appearance of a corresponding bifurcation set. In order to analyze this assumption, we have generalized 


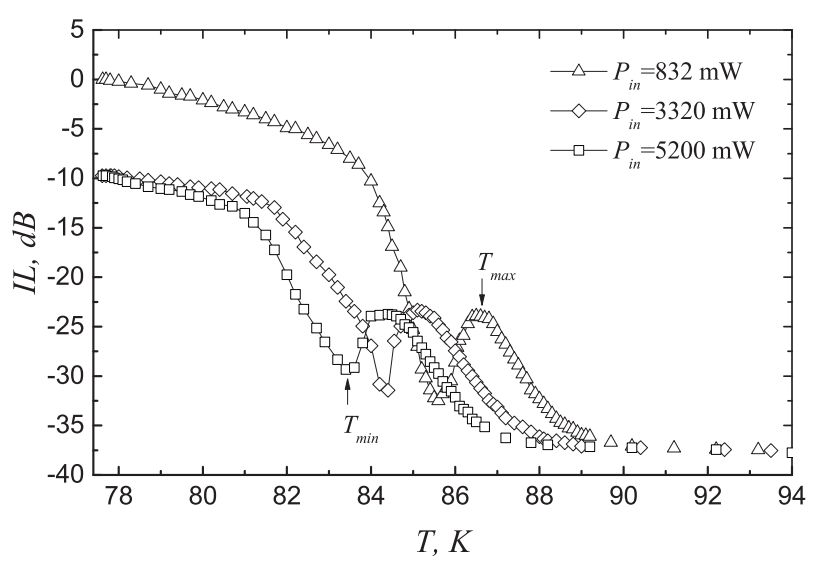

Fig. 3. Formation of a "fold" at the two-dimensional surface $I L\left(P_{\mathrm{in}}, T\right)$ of possible CPW states. The HTSC film thickness is $75 \mathrm{~nm}[6]$

the available phenomenological model of a microwave nonlinear HTSC-based transmission line [8-10] to the case of non-zero direct current $I_{d c}$ and have considered the next term in the series expansion of the nonlinear dependence of the resistance on the total current.

\section{Phenomenological Model of the Current Flow Through an HTSC Waveguide}

Parameters of a nonlinear waveguide based on HTSCs are governed by the nonlinearity in the physical properties of an HTSC epitaxial film. The main origins of this nonlinearity can be as follows [12]:

- the current dependence of the superconducting charge carrier concentration in accordance to the Ginzburg-Landau equations [2] and

- excitation and motion of Abrikosov vortices [13].

The elaboration of a physical model that would take all those effects into account is a complicated task that has not been completely resolved till now. In this connection, to explain the available experimental data, a phenomenological model of nonlinearity in the HTSC properties is used, as a rule. In particular, as was shown in work [10], the phenomenological description of nonlinearities in the superconducting film properties brings about numerical results that are in good agreement with experimental data. Furthermore, the nonlinearity of the inductive impedance component was additionally taken into account in work [8].

\section{4}

The well-known phenomenological model is based on the account for only the first non-zero term in the expansion of the nonlinear dependence of the linear resistance on the current [10]. Since the properties of the HTSC film are isotropic, this term is a quadratic one:

$$
\begin{aligned}
& R_{1}(t)=\sum_{n=0}^{\infty} a_{n}[I(t)]^{2 n} \approx a_{0}+a_{1}[I(t)]^{2}= \\
& =R_{1.1}\left[1+\frac{I(t)^{2}}{I_{0 R}}\right] .
\end{aligned}
$$

Here, $R_{1.1}$ is the initial (in the absence of a current) linear resistance of a transmission line, which depends on the effective surface resistance of a superconductor, $R_{s}$, and the geometric dimensions of the waveguide cross-section. Note that Eq. (1) does not contain persistent components. Strictly speaking, this is true, if the characteristic relaxation time of a superconducting film state is much shorter than the period of microwave oscillations.

When analyzing the model, telegraph equations for a waveguide line were solved using the harmonic balance method [10]. Since nonlinearity (1) leads to an anharmonic solution, the latter can be represented as the sum of harmonics. The following equation was obtained for the first harmonic of the current amplitude $I_{m}(x)$ :

$\frac{d I_{m}(x)}{d x}+\alpha I_{m}(x)\left[1+\frac{3}{4} \frac{I_{m}^{2}(x)}{I_{0 R}^{2}}\right]=0$,

where $\alpha=R_{1} /\left(2 Z_{0}\right)$ and $Z_{0}=\sqrt{L_{1} / C_{1}}$ are the propagation constant and the wave impedance of the line, respectively, for the quasi- $T$ waves in the transmission line $\left(Z_{0}=50 \Omega\right.$ for the examined waveguides). Equation (2) has an analytic solution

$I_{m}^{2}(x)=I_{m}^{2}(0) \frac{\exp (-2 \alpha x)}{1+\frac{3}{4} \frac{I_{m}^{2}(0)}{I_{0}^{2}}[1-\exp (-2 \alpha x)]}$.

Then, for a waveguide line of the length $l$, the ratio between the input and output powers for the first current harmonic looks like

$I L=10 \lg \left(\frac{P_{\text {out }}}{P_{\text {in }}}\right)=-\frac{10}{\ln 10} \times$
$\times\left\{2 \alpha l+\ln \left[1+\frac{3}{4} \frac{P_{\text {in }}}{P_{0}}[1-\exp (-2 \alpha l)]\right]\right\}$

ISSN 2071-0194. Ukr. J. Phys. 2019. Vol. 64, No. 10 
where $P_{0}=\frac{1}{2} I_{0 R}^{2} Z_{0}$, and $I_{0 R}$ is a phenomenological parameter.

The experimental results obtained in works [8-10] confirm the adequacy of the model and the validity of formula (4) for temperatures well below $T_{\mathrm{c}}$. But the results of our studies (see Fig. 3 in work [5]) do not agree with this formula in the general case. In particular, at temperatures close to the critical one, the value of $I L$ may decrease with the growth of $P_{\text {in }}$, what is not allowed in relation (4).

For the explanation of the "fold" effect in the experimental dependence $I L(T)$ (see Fig. 3 in work [6]), a number of physical mechanisms were proposed. They can be associated with both the microscopic phenomena in HTSCs and the macroscopic properties of a waveguide [8]. However, when considering the phenomenological model, we are not interested in the physical nature of a nonlinearity, but in its mathematical representation.

To achieve the agreement with the experiment, either of two approximations accepted in works [8-10] should be rejected. This is either the assumption that the properties of the HTSC film are not persistent at frequencies below $10 \mathrm{GHz}$ or the assumption that the series expansion in Eq. (1) can be truncated after the first non-zero term. Let us analyze the both possibilities.

\subsection{Persistence of HTSC properties and its effect on the experimental dependence $\boldsymbol{P}_{\text {out }}\left(\boldsymbol{P}_{\mathrm{in}}\right)$}

In order to accurately study and simulate the persistence of the electrophysical properties of HTSCs, relaxation phenomena have to be taken into account making use of their adequate physical description. However, the final effect of this procedure results in the introduction of an HTSC "memory function" $f_{R}\left(t-t^{\prime}\right)$, which is a kernel of the following convolution integral transformation used to specify the linear resistance values:

$R_{1}^{*}(t)=\int_{-\infty}^{t} f_{R}\left(t-t^{\prime}\right) R_{1}\left(t^{\prime}\right) d t^{\prime}$.

In order to change to the frequency representation of the signal, it is necessary to formally extend the interval of the memory function definition onto the whole time axis, $t^{\prime} \in(-\infty ; \infty)$, and perform the
Fourier transformation. The result looks like

$\widetilde{R_{1}^{*}}(\omega)=\widetilde{f_{R}}(\omega) \widetilde{R_{1}}(\omega)$.

The dependence $R_{1}(t)$ is calculated using formula (1) and has a discrete spectrum $\widetilde{R_{1}}(\omega)$. At the same time, the spectrum of the memory function $\widetilde{f_{R}}(\omega)$ is continuous in the general case. Therefore, the dependence $R_{1}^{*}(t)$ obtained from $\widetilde{f_{R}}(\omega)$ as the inverse transform has a continuous spectrum. Therefore, the experimentally measured $I L(\omega)$ spectrum becomes smeared. Hence, the persistence effect on the experimental results for the first current harmonic is reduced to the multiplication of the first harmonic of the non-persistent dependence $R_{1}(t)$ by a certain complex-valued phenomenological parameter $\widetilde{f_{R 0}}$. In order to determine this parameter accurately, it is necessary to specify (or experimentally measure) the HTSC "memory function" $f_{R}\left(t-t^{\prime}\right)$.

The dependence $R_{1}(t)$ calculated by formula (1) includes the parameter $f_{R 0}$ and may depend both on the HTSC temperature, because the relaxation processes run at different rates at different temperatures, and on the current frequency. Formally, the parameter $f_{R 0}$ can be represented as an additional inductive component of the resistance. However, the analysis of the conditions, under which its introduction can lead to observed experimental anomalies, requires a more detailed consideration and goes beyond the scope of this work.

\subsection{Account for the next term in the series expansion of HTSC properties in the current}

The inclusion of the fourth-oreder term into series expansion (1) gives rise to the following approximation for the linear resistance:

$R_{1}(t) \approx R_{1,1}\left[1-\frac{I(t)^{2}}{I_{0 R}^{2}}+\frac{I(t)^{4}}{I_{1 R}^{4}}\right]$.

The signs of terms in this approximation were chosen to provide a possibility for the linear resistance to have a non-monotonic dependence on the input microwave power and to satisfy the asymptotic limit of $R_{1}\left(P_{\text {in }}\right)$. An example of this dependence is shown in Fig. 4.

After substituting formula (7) into the system of telegraph equations and solving it using the method 


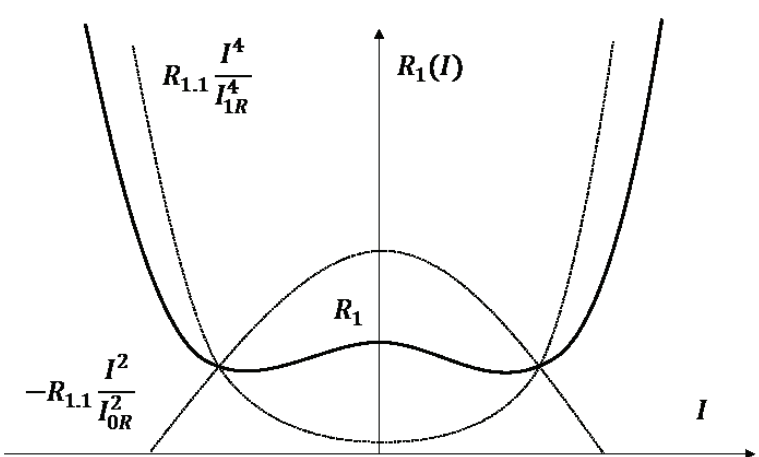

Fig. 4. Approximation of line resistance making allowance for the fourth-order current term in the series expansion

of harmonic balance for the first (fundamental) harmonic of a signal, we obtain the first-order differential equation

$-\frac{1}{U} \frac{d U}{d x^{\prime}}=1-\frac{3}{4} U+\frac{5}{8} \theta U^{2}$

where

$\ln U+\frac{3}{8} \frac{1}{\sqrt{\frac{5}{2} \vartheta-\frac{9}{16}}} \arctan \left(\frac{\frac{5}{4} \vartheta U-\frac{3}{4}}{\sqrt{\frac{5}{2} \vartheta-\frac{9}{16}}}\right)-$
$-\frac{1}{2} \ln \left(1-\frac{3}{4} U+\frac{5}{8} \vartheta U^{2}\right)+C=-\frac{5}{8} \vartheta x^{\prime}$.

This formula makes it possible to construct the dependence of the quantity $I L=4.34 \ln \left(P_{\text {out }} / P_{\text {in }}\right)$ on the parameters $P_{\text {in }} / P_{0}, \alpha$, and $\vartheta$ and compare it with experimental data [5].

\section{Model Adaptation}

\section{in the Case of Non-Zero Direct Current}

If the direct current component differs from zero, a solution of the telegraph equations for the first current harmonic should be sought in the form

$I(x, t)=I_{m}(x) \cos (\omega t-\beta x)+I_{\mathrm{dc}}$.

Now, instead of Eq. (8), we obtain the equation for the coordinate dependence of the amplitude of the first current harmonic, $I_{m}(x)$ :

$$
\begin{aligned}
& -\frac{1}{U} d U d x^{\prime}=2 \alpha\left[\left(1-3 \frac{I_{\mathrm{dc}}^{2}}{I_{0 R}^{2}}+5 \vartheta \frac{I_{\mathrm{dc}}^{4}}{I_{0 R}^{4}}\right)-\right. \\
& \left.-\frac{3}{4} U\left(1-10 \vartheta \frac{I_{\mathrm{dc}}^{2}}{I_{0 R}^{2}}\right)+\frac{5}{8} \vartheta U^{2}\right] .
\end{aligned}
$$

The change of the variable $U$ and the parameters $\alpha$ and $\vartheta$,

$$
\begin{aligned}
& \alpha^{*}=\alpha\left(1-3 \frac{I_{d c}^{2}}{I_{0 R}^{2}}+5 \vartheta \frac{I_{d c}^{4}}{I_{0 R}^{4}}\right) ; \\
& U^{*}=\frac{\left(I_{m}^{*}\right)^{2}}{I_{0 R}^{2}}=\frac{I_{m}^{2}}{I_{0 R}^{2}}\left(1-10 \vartheta \frac{I_{d c}^{2}}{I_{0 R}^{2}}\right) / \\
& /\left(1-3 \frac{I_{d c}^{2}}{I_{0 R}^{2}}+5 \vartheta \frac{I_{d c}^{4}}{I_{0 R}^{4}}\right) ; \\
& I_{m}^{*}=I_{m} \sqrt{\left(1-10 \vartheta \frac{I_{d c}^{2}}{I_{0 R}^{2}}\right) /\left(1-3 \frac{I_{d c}^{2}}{I_{0 R}^{2}}+5 \vartheta \frac{I_{d c}^{4}}{I_{0 R}^{4}}\right)} \\
& \vartheta^{*}=\vartheta\left(1-3 \frac{I_{d c}^{2}}{I_{0 R}^{2}}+5 \vartheta \frac{I_{d c}^{4}}{I_{0 R}^{4}}\right) /\left(1-10 \vartheta \frac{I_{d c}^{2}}{I_{0 R}^{2}}\right)^{2}- \\
& -\frac{1}{U^{*}} \frac{\partial U^{*}}{\partial\left(2 \alpha^{*} x\right)}=1-\frac{3}{4} U^{*}+\frac{5}{8} \vartheta^{*} U^{* 2}
\end{aligned}
$$

makes it possible to reduce Eq. (11) to Eq. (8) and, hence, to use the obtained analytic solution (9).

The change of the variables $U$ (the corresponding change of the quantity $I_{m}^{2}$ ) and $\alpha$ (the corresponding change of the quantity $I_{0 R}^{2}$ ) can be simulated by nonlinearly transforming the given parameters of the dependence $T^{*}=f_{T}(T)$ and parameters of the input microwave power $P_{\text {in }}^{*}=f_{P}\left(P_{\text {in }}\right)$. In this case, the response surface $I L\left(P_{\mathrm{in}}, T\right)$, which was determined experimentally, will retain its shape in the new control parameters, $I L\left(P_{\mathrm{in}}^{*}, T^{*}\right)$. No conditions for invoking a "catastrophe" will arise at that.

However, the change of the model parameter $\vartheta$ cannot be reduced to a nonlinear rescaling of axes for the experimentally determined two-dimensional surface $I L\left(T, P_{\text {in }}\right)$, as was done in the case of model (1). This rescaling deforms the very surface of the "fold" obtained in the absence of the $I_{d c}$ component. Therefore, below, we will analyze a numerical solution of Eq. (12) and show that the "catastrophe" effect may emerge at some values of input parameters (Fig. 2).

\section{Possibility of the "Catastrophe" Effect for a Non-Zero Direct Current Component}

In order to simplify the analysis of solution (12), let us introduce the notation $U_{d c}=I_{d c}^{2} / I_{0 R}^{2}$. Provided that the condition $\frac{9}{16}-\frac{5}{2} \vartheta^{*}>0$ or

$$
\frac{9}{16}\left(1-10 U_{\mathrm{dc}}\right)^{2}>\frac{5}{2} \vartheta\left(1-3 U_{\mathrm{dc}}+5 \vartheta U_{\mathrm{dc}}^{2}\right)
$$

is satisfied, the right-hand side of Eq. (12), which is regarded as a quadratic trinomial in $U^{*}$, has real 


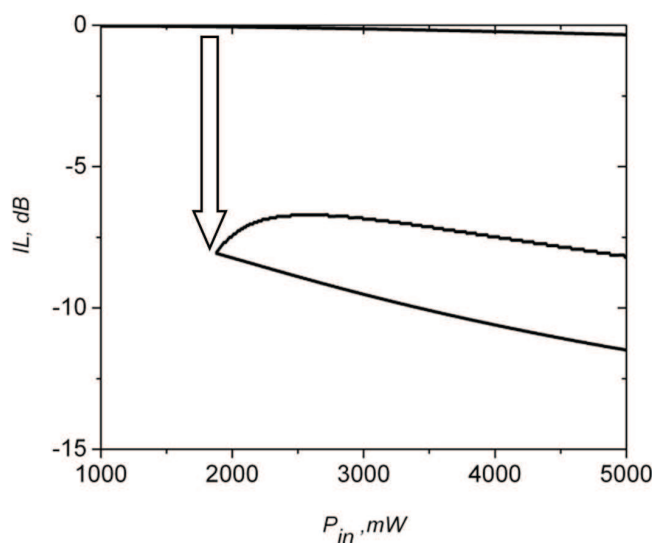

Fig. 5. Emergence of the "catastrophe" effect at a gradual increase of the $I_{d c}$ current in the framework of model (11), (12)

$\operatorname{roots} U_{1,2}^{*}$. If, additionally,

$1-3 U_{\mathrm{dc}}+5 \vartheta U_{\mathrm{dc}}^{2}>0$,

the both roots are positive and may fall within the interval of possible values for $U^{*}(x)$. They are equilibrium points of the quantity $U^{*}$. The smaller roots correspond to the unstable equilibrium of the function $U^{*}(x)$, and the larger root to the stable one.

When the direct current amplitude or the temperature are gradually changed in the experiment, the parameters $\vartheta$ and $U_{d c}$ in Eqs. (13) and (14) also change gradually. As a result, if the input microwave power $P_{\text {in }}$ is constant, the corresponding value $U^{*}(0)$ turns out in different regions of the $U^{*}$-scale with respect to the unstable equilibrium point $U_{1}^{*}$. Accordingly, the $U^{*}(l)$ value corresponding to $P_{\text {out }}$ changes in a jumplike manner.

To simulate this "catastrophe" effect, a program for the numerical solution of Eq. (12) at various values of control parameters was written. In Fig. 5, an example of the theoretical dependence $I L\left(I_{d c}\right)$ calculated for the given parameters $T$ and $P_{\text {in }}$ is shown. One can see that the absolute value $|I L|$ abruptly increases at $I_{d c} \approx 65 \mathrm{~mA}$, which is similar to experimental dependences (Fig. 6). A further growth of $I_{d c}$ leads to the strong heating of a waveguide and cannot be adequately described in the framework of the proposed model.

We also calculated the dependence $I L\left(P_{\text {in }}\right)$ for the given values of $T$ and $I_{d c}$ (Fig. 7). One can see that, at the input power values $P_{\text {in }}>1821 \mathrm{~mW}$, there arises a bifurcation set of parameters, for which a "catas-

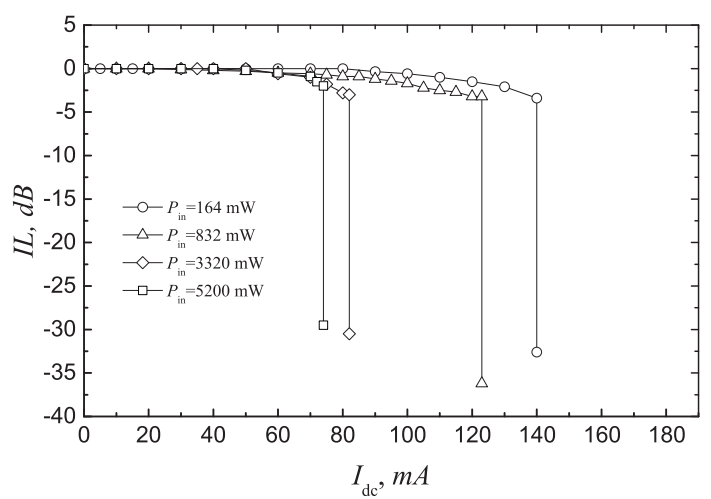

Fig. 6. Experimental dependences of the power losses on the direct current magnitude for a CPW based on an HTSC film $75 \mathrm{~nm}$ in thickness for various values of the input power $P_{\text {in }}$

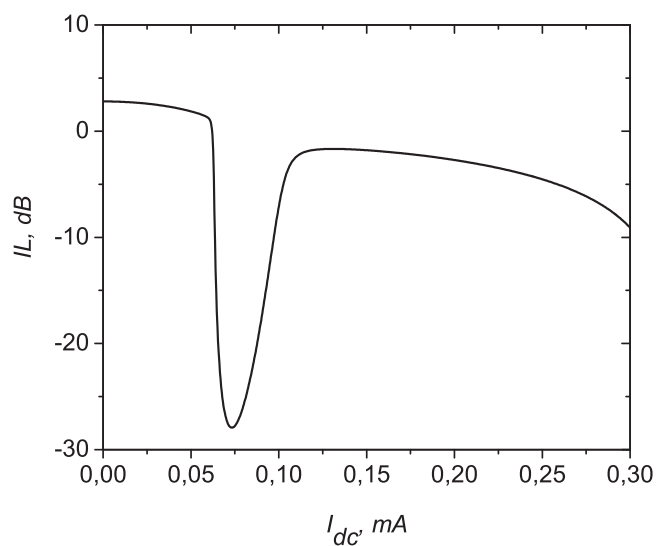

Fig. 7. Emergence of a bifurcation set and a possible "catastrophe" at a gradual increase of the input microwave power in the framework of the model concerned

trophe" - a jump-like transition into the steady solution - is possible. Note that Figs. 5 and 7 only illustrate some capabilities of the developed model. In order to analyze the numerical correspondence of the presented calculation results to the experiment, the temperature dependences of the model parameters have to be found. This task is planned to be performed in the future.

\section{Conclusions}

In this work, the nonlinear phenomenological theory of the HTSC-based transmission line has been generalized to the case of the non-zero direct current. The proposed model allows the following conclusions to be made.

- The account for only the quadratic term in the expansion of the nonlinear dependence of the linear 
impedance on the current cannot explain the experimental data obtained for various values of the input microwave signal power.

- The account for the next (fourth-order) term in the expansion of the nonlinear dependence of the linear impedance on the current leads to the appearance of an additional (quadratic) term in Eq. (8) for the coordinate dependence of the microwave signal amplitude. However, this amendment does not lead to the "catastrophe", a jump-like change in the properties of the transmission line, when the control parameters are varied gradually.

- An additional constant current flowing through the transmission line may induce the appearance of a bifurcation region in the control parameter space. As a result, there emerges a possibility for the waveguide to drastically change its properties at the boundary of this region. A qualitative correspondence between the results of the generalized phenomenological model and the data of experimental studies is obtained.

1. A.Ya. Kirichenko, V.B. Kosmyna, A.B. Levin, N.T. Cherpak. Possible stimulation of superconductivity in $\mathrm{Bi}-\mathrm{Sr}-$ $\mathrm{Ca}-\mathrm{Cu}-\mathrm{O}$ by electromagnetic radiation. JETP Lett. 50, 290 (1989).

2. J.H. Oates, R.T. Shin, D.E. Oates, M.J. Tsuk, P.P. Nguyn. A nonlinear transmission line model for superconducting stripline resonators. IEEE Trans. Appl. Supercond. 3, 17 (1993).

3. M. Hein. High-Temperature-Superconductor Thin Films at Microwave Frequencies (Springer, 1999).

4. J.C. Booth, D.A. Rudman, R.H. Ono. A self-attenuating superconducting transmission line for use as a microwave power limiter. IEEE Trans. Appl. Supercond. 13, 305 (2003).

5. N. Cherpak, A. Lavrinovich, A. Gubin, S. Vitusevich. Directcurrent-assisted microwave quenching of $\mathrm{YBa}_{2} \mathrm{Cu}_{3} \mathrm{O}_{7-\delta}$ coplanar waveguide to a highly dissipative state. Appl. Phys. Lett. 105, 022601 (2014).

6. N. Cherpak, A. Lavrinovich, A. Gubin, S. Vitusevich. Microwave quenching in DC-biased coplanar waveguide based on $\mathrm{YBa}_{2} \mathrm{Cu}_{3} \mathrm{O}_{7-\delta}$ thin film. IEEE Trans. Appl. Supercond. 26, 1501204 (2016).

7. R. Wordenweber, E. Hollmann, J. Schubert, R. Kutzner, G. Panaitov. Regimes of flux transport at microwave fre- quencies in nanostructured high- $T_{\mathrm{c}}$ films. Phys. Rev. B 85, 064503 (2012).

8. N.T. Cherpak, A.A. Lavrinovich, A.A. Kalenyuk, et al. DC-biased coplanar waveguide on the basis of highTc superconducting thin film with nonlinear impedance. Telecommun. Radio Engin. 69, 1357 (2010).

9. A.A. Lavrinovich, E.V. Khramota, N.T. Cherpak. Investigation of the superconducting microwave transmission line in strong electromagnetic fields. Telecommun. Radio Engin. 68, 1741 (2009).

10. I.B. Vendik, O.G. Vendik, D. Kaparkov. High Temperature Superconductor Devices for Microwave Signal Processing. Part II. Superconducting Microwave Circuits (Skladen', 1997).

11. T. Poston, I. Stewart. Catastrophe: Theory and Its Applications (Dover, 1998).

12. O.G. Vendik, I.B. Vendik, T.B. Samoilova. Nonlinearity of superconducting transmission line and microstrip resonator. IEEE Trans. Microwave Theory 45, 173 (1997).

13. C.C. Chin, D.E. Oates, G. Dresselhaus, M.S. Dresselhaus. Nonlinear electrodynamics of superconducting $\mathrm{NbN}$ and $\mathrm{Nb}$ thin films at microwave frequencies. Phys. Rev. B 45, 4788 (1992)

Received 22.01.09

С.I. Мелъник, С.С. Мелъник,

О.А. Лавринович, М.Т. Черпак

ДО ФЕНОМЕНОЛОГІЧНОЇ ТЕОРІЇ

ЛАВИНОПОДІБНОГО ЕФЕКТУ В МІКРОХВИЛЬОВІЙ НЕЛІНІЙНІЙ ВТНП ЛІНІЇ ПЕРЕДАЧІ

З ПОСТІЙНИМ СТРУМОМ

$\mathrm{P}$ е $з$ ю м е

У даній роботі пропонується феноменологічна теоретична модель лавиноподібного переходу мікрохвильової нелінійної ВТНП лінії передачі в дисипативний стан. Цей ефект спостерігався авторами в лінії передачі з постійним струмом. Запропонована модель узагальнює відому феноменологічну модель нелінійної ВТНП лінії передачі при впливі на неї постійного струму. Характер поведінки залежностей мікрохвильових втрат, що досліджуються, дозволяє розглядати стрибкоподібні зміни властивостей нелінійної ВТНП лінії передачі, як "катастрофу" типу "складки" і використовувати методологічний і математичний апарат теорії "катастроф" для пояснення отриманих і передбачення нових результатів. 